\title{
Los cinco: ¿agentes, espías o héroes? Hacia un análisis crítico del discurso de \#5DaysForTheFive ${ }^{1}$
}

\author{
Enrique Pérez-Fumero² \\ Yamile Haber-Guerra ${ }^{3}$ \\ Franky Sánchez-Barriel ${ }^{4}$ \\ Aimé Sosa-Pompa ${ }^{5}$
}

Recibido: 2014-08-28

Enviado a pares: 2014-09-01
Aprobado por pares: 2014-11-27

Aceptado: 2014-12-05

DOI: 10.5294/pacla.2015.18.3.10

Para citar este artículo / To reference this article / Para citar este artigo

Pérez-Fumero, E., Haber-Guerra, Y., Sánchez- Barriel, F. \& Sosa-Pompa, A. (Septiembre de 2015). Los cinco: ¿agentes, espías o héroes? Hacia un análisis crítico del discurso de \#5DaysForTheFive. Palabra Clave, 18(3), 859-888. DOI: 10.5294/pacla.2015.18.3.10

\section{Resumen}

Aquí presentamos un examen sobre al tratamiento discursivo concerniente al caso de los cinco cubanos Gerardo Hernández, Antonio Guerrero, Ramón Labañino, Fernando González y René González, quienes estuvieron presos en Estados Unidos, con particular interés en la exploración de las zonas de dominación, poder e ideología socializadas en Twitter. A partir de la relación entre lenguaje, discurso periodístico, conocimiento y las perspectivas del análisis crítico del discurso (ACD), como los estudios cognitivos y el análisis de discurso orientado textualmente, elaboramos un instrumento metodológico para estudiar una muestra de tuits que contienen la etiqueta \#5DaysForTheFive, dentro del contexto de la Tercera Jornada 5 días por los 5, celebrada en Washington del 4 al 11 de junio de 2014. En el artículo

\footnotetext{
En todos los tuits se ha respetado la grafía original.

Universidad de Oriente, Cuba. enriquep@rect.uo.edu.cu

Universidad de Oriente, Cuba. yhaber@enet.cu

DATYS Tecnología \& Sistemas, Cuba. franky.sanchez@datys.cu

Universidad de Oriente, Cuba. aimucha@rsiboney.icrt.cu
} 
se identifican las formas en que los abusos de poder sobre el caso tienen lugar gracias a su legitimación desde el discurso y la respuesta ideológica que dan los usuarios y seguidores de la red de microblogging.

\section{Palabras clave}

Análisis, discurso, estudios sociales, análisis del discurso, Twitter, poder, conocimiento, \#5DaysForTheFive (Fuente: Tesauro de la Unesco).

\section{The Five: Agents, Spies or Heroes? Towards a Critical Discourse Analysis of \# 5DaysForTheFive}

\section{Abstract}

Here is an examination of the discursive treatment concerning the case of five Cubans Gerardo Hernandez, Antonio Guerrero, Ramón Labañino, Fernando Gonzalez and Rene Gonzalez, who were imprisoned in the United States, with particular interest in exploring areas of domination, power and ideology socialized in Twitter. From the relationship between language, journalistic discourse, knowledge and perspectives of the ACD, such as cognitive studies and textually oriented discourse analysis, we developed a methodological tool to study a sample of tweets containing the hashtag \# 5DaysForTheFive, within the context Third 5 days for The Cuban 5, held in Washington, D.C. from June 4 to 11, 2014. In the article identified the ways in which abuses of power on the case take place are identified, thanks to its legitimacy from discourse and the ideological response that users and followers give from the microblogging network.

\section{Keywords}

Analysis, discourse, social studies, discourse analysis, Twitter, power, knowledge, \# 5DaysForTheFive (Source: Unesco Thesaurus). 


\section{Os cinco: agentes, espiões ou heróis? Rumo a uma análise crítica do discurso de \#5DaysForTheFive}

\section{Resumo}

Aqui apresentamos um exame sobre o tratamento discursivo concernente ao caso dos cinco cubanos Gerardo Hernández, Antonio Guerrero, Ramón Labañino, Fernando González e René González, que estiveram presos nos Estados Unidos, com particular interesse na exploração das áreas de dominação, poder e ideologia socializadas no Twitter. A partir da relação entre linguagem, discurso jornalístico, conhecimento e as perspectivas da análise crítica do discurso, como os estudos cognitivos e a análise do discurso orientado textualmente, elaboramos um instrumento metodológico para estudar uma amostra de tweets que contêm a etiqueta \#5DaysForTheFive, dentro do contexto da Terceira Jornada 5 dias pelos 5, celebrada em Washington do dia 4 a 11 de junho de 2014. No artigo, identificam-se as formas como os abusos de poder sobre o caso acontecem graças a sua legitimação a partir do discurso e da resposta ideológica que dão os usuários e seguidores da rede de microblogging.

\section{Palavras-chave}

Análise, discurso, estudos sociais, análise do discurso, Twitter, poder, conhecimento, \#5DaysForTheFive (Fonte: Tesauro da Unesco). 


\section{Introducción}

“¿Cinco héroes o cinco espías? La verdad sobre el caso 'Avispa' contada por uno de sus protagonistas”, apunta el profesor Edgerton Ivor Levy (2013) en el blog del periodista Carlos Alberto Montaner. "Los Cinco Cubanos estaban combatiendo el terrorismo. ¿Por qué los encarcelamos?”, se puede leer en una nota del diario norteamericano The Washington Post, donde su autor, el investigador y periodista canadiense Stephen Kimber (2013), reconoce que "estos agentes serían héroes norteamericanos hoy". "Regresa a La Habana uno de los cinco espías cubanos presos en EE UU”, encabeza la noticia en el diario El País, de España.

Asistimos al consumo de diversos términos, agendas, encuadres noticiosos y contextos lingüísticos específicos utilizados por los medios para explicar la realidad de un conflicto político particularmente complejo entre Cuba y Estados Unidos.

Desde que comenzara, en 1998, la campaña por la liberación de Gerardo Hernández, Antonio Guerrero, Ramón Labañino, Fernando González y René González, la prensa cubana los nombró "héroes prisioneros del imperio" (Falcón, 2014), además de llamarlos "luchadores, antiterroristas, o pacifistas". Sin embargo, ¿a quiénes -lectores, receptores, destinatarios, enunciatarios, prosumidores y @usuarios- se ha tratado de socializar tal enunciado?

Luego de 15 años, todavía nos encontramos ante un evento político y discursivo internacional que demandó la negociación no solo de los presidentes norteamericano y cubano, como ocurrió el 17 de diciembre de 2014, cuando Barack Obama y Raúl Castro comparecieron ante las cámaras y anunciaron el restablecimiento de las relaciones diplomáticas, sino de otras élites de poder. Precisamente, una de estas acciones tuvo lugar en Washington, D. C., del 4 al 11 de junio de 2014, cuando el Comité Internacional por la Libertad de los 5 Cubanos con base en Estados Unidos organizó la Tercera Jornada 5 días por los 5. Esta iniciativa también se desarrolló desde Twitter, donde millones de personas se 
sumaron con el hashtag ${ }^{6}$ \#5 DaysForTheFive, y convirtieron el hecho en un trending topic.

Dado que la solidaridad persistió a través de las acciones de los comités y con el regreso a Cuba de René González y Fernando González luego de cumplir sendas condenas íntegras, y de Gerardo Hernández, Antonio Guerrero y Ramón Labañino el 17 de diciembre de 2014, este estudio está dirigido a demostrar y a desvelar las relaciones de poder, ideología y dominación reproducidas en el discurso sobre los cinco cubanos. Una segunda problemática anima a desentrañar, desde un texto de 140 caracteres o más, los signos empleados y sus significados para la campaña.

\section{Twitter como evento comunicativo de poder y dominación}

El estudio de las complejas estructuras inherentes al discurso de las noticias periodísticas ha servido de caldo de cultivo para aproximarse a los actos comunicativos en la era de los blogs y otras redes sociales online (Flores 2012; Merodio, 2010; Orihuela, 2011; Conde \& Moreno, 2012; Iluminati $\mathrm{Lab}, 2012)$. Este nuevo discurso periodístico tiene su origen en la semiosis que se establece entre los lenguajes y reconoce, de acuerdo con la investigadora Yamile Haber, "la figura pragmalingüística del enunciatario, como destinatario del mensaje que al propio tiempo enuncia y da sentido; la del cibernauta prosumidor, que al consumir mientras se informa, produce texto, o la del productor usuario, producer, en inglés, todas pragmáticamente emparentadas" (2014, p. 4). La red de microblogging Twitter es un ejemplo práctico, dado que quienes la usan "son personas conversando con personas y creando vínculos con personas. Gente hablando, escuchando y respondiendo. Twitter es personal y es comunidad" (Orihuela, 2011, p.78).

Una muestra de esos actos conversacionales se evidenció cuando los@usuarios y sus @seguidores utilizaron la red como plataforma política y noticiosa al participar de hechos como la Primavera Árabe y el tercer

6 En adelante, reconózcanse indistintamente los términos hashtag, almohadilla, o etiqueta para identificar la cadena de caracteres formada por una o varias palabras concatenadas y precedidas en servicios web tales como Twitter, FriendFeed, Instagram o Google+ por el símbolo de numeral (\#). 
aniversario del movimiento \#15M (Público, 2014) u opinar sobre el conflicto \#ucraniacrisis.

Sin embargo, cada persona que participa activa y creativamente en esta plataforma demuestra un tipo de conocimiento (ideología) y socializa otra manera (poder) de interpretar los asuntos políticos. Alrededor de la actividad con la almohadilla \#5DaysForTheFive, @PlacidoMax ${ }^{7}$ escribió: "Afirman que \#EEUU debe encontrar solución política en caso de Los Cinco \#5DaysForThe5 \#FreeTheFive \#Cuba”. En cambio, @jdanielferrer manifestó: “\#Cuba. Todo cubano adulto que desee una Cuba Libre, democrática y junta puede unirse a \#UNPACU \#5DaysForThe5”.

Aquí queda demostrada la capacidad del discurso a través de cualquiera de sus formas (texto, lenguaje, imágenes, conversaciones, etc.) para reproducir y legitimar las ideologías de los grupos elitistas, que dependen de estas formas simbólicas para socializarse. El panorama actual de los social media hace posible, en consecuencia, la investigación de cómo los grupos dominados responden discursivamente a los abusos de poder, siempre respetando sus afirmaciones y el contexto sociocultural y político en que fueron hechas. ${ }^{8}$ Justamente por ello y porque Twitter, dada su naturaleza pública, es utilizado como "si se hablara en voz alta para que todo el mundo lo oiga” (Luz, Fernández \& Moro, 2011, p.12), nuestro análisis no falta a la ética ni en el sentido periodístico más clásico (García Luis, 2013) ni en el científico-comunicológico (Martín Serrano, 2013). Somos red y la construcción de nuestra identidad digital "seguirá un proceso lento, torpe y salpicado por momentos de la más deleble retórica” (Fumero, 2011, p. 45). Hoy en día, la conversación en red deviene un objeto importante para medir el estado de la opinión pública, sobre todo en asuntos políticos.

Si asumimos como discurso periodístico el evento comunicativo desarrollado desde el surgimiento de la etiqueta \#5DaysForTheFive, tenemos

7 El uso aquí de las identidades de usuarios (personas, grupos o instituciones), que en el caso de Twitter van antecedidas con el símbolo de @, es puramente con fines académicos.

8 La reutilización de un texto, canción, post o un simple tuit depende de los términos y las condiciones online que haya utilizado el autor para distribuir esa pieza (algo que la mayoría no hace) y que se convierte en un contrato firmado toda vez que pulse el botón "aceptar" (Fumero, 2011, p. 45). 
que integrar los roles desempeñados por quienes escriben, tuitean, opinan y retuitean mensajes en paralelo a las situaciones que rodearon esos actos comunicativos, como la proxemia, la comunicación intercultural, el tiempo que duró la campaña y otras características sociales que distinguen el contexto y el conocimiento precedente. A continuación explicaremos, desde una perspectiva transdisciplinar, la participación del lenguaje, el discurso y el conocimiento para luego relacionar el análisis crítico del discurso (ACD) y dos de sus enfoques más pertinentes para esta investigación: los estudios sociocognitivos y el análisis de discurso orientado textualmente.

\section{Lenguaje, conocimiento y discurso.} Hacia una búsqueda del análisis crítico del discurso El entendimiento, la comprensión y la socialización del discurso periodístico jerarquizado por los medios parten de reconocer el lugar que ocupa el lenguaje en la vida y el imaginario de las personas. Hay que tener en cuenta los tratados de la teoría de la lingüística moderna, a través de la concepción de sistemas de signos empleada por el estudioso suizo Ferdinand de Saussure. El lingüista danés Louis Hjelmslev (1943) definía los presupuestos dellenguaje, los signos y la sintaxis de la frase como "una entidad autónoma de dependencias internas" en su libro Prolegomena to a Theory of Language. Pero la jerarquía de las expresiones, más allá de los sistemas articulados fonológico, lexical y sintáctico-, se amplía cuando trasciende estos niveles para convertirse en frase o enunciado, es decir, palabra o discurso.

Es imprescindible que las personas posean algún tipo de conocimiento general, gramatical, político o lingüístico para consumir y reproducir las oraciones, los textos y los significados, visibles o no, en que se presenta el discurso actual. Estos conocimientos — la mayoría aportados por instituciones de poder simbólico y epistémico como las universidades, las iglesias, las comunidades científicas, los tribunales y los medios- son almacenados en la memoria a largo plazo.

Sin embargo, cada hecho que adquiere renombre en la prensa es objeto de múltiples interpretaciones por quienes crean modelos mentales o representaciones subjetivas de los hechos y las situaciones para entender 
la naturaleza de los textos. En resumen, y siguiendo al investigador Teun A. van Dijk, la comprensión del discurso se realiza por "un modelo mental, y creamos nuestro propio modelo personal de un texto aplicando nuestro conocimiento general, socioculturalmente compartido" (2010, p. 172). A su vez, el especialista holandés también denota la importancia de los "modelos contextuales" como otra forma de construir y gestionar conocimientos, visto que las personas, los frames y los medios adaptan sus maneras de hablar y de escribir a determinadas situaciones lingüísticas.

El discurso es un acto o evento comunicativo en el que participan diversos actores con igual número de roles en una situación específica y determinado por otras características contextuales. Es un resultado "verbal, oral o escrito, del acto comunicativo, cuya unidad de uso (lenguaje) pertenece a la unidad teórica abstracta (texto) dentro del conocimiento lingüístico" (Londoño \& Frías, 2011, p. 103).

Una revisión de los diversos enfoques que se han encargado de la relación entre el lenguaje y el discurso retrotrae los aportes de la psicología cognitiva (Turner, 1996) y la psicolingüística (Traxler \& Gernsbacher, 2006), la semiótica (Eco, 1973, 1991) y el análisis conversacional (Sacks, Schegloff \& Jefferson, 1974).

Teóricamente, el lenguaje comparte funciones como "medio de comunicación de sentido social y como almacén de conocimientos" (Herzog et al., 2006, p. 5), pero la comunicación en un sentido amplio analiza los actos desde el punto de vista del lenguaje como desde el punto de vista social, enmarcados en procesos discursivos. Esta interpretación dualista que relaciona los estudios lingüísticos con los discursivos y reconoce la importancia de actores, prácticas no discursivas e instituciones para el proceso comunicativo ha constituido uno de los objetos de estudio de la sociología del conocimiento (Keller, 2005). Siguiendo a Keller en su enfoque de análisis del discurso basado en la sociología del conocimiento (ADSC), e interpretándolo desde las redes sociales como Twitter, nos podríamos hacer las siguientes preguntas: 
- ¿ ¿Cuál es el conocimiento que se socializa en un texto de 140 caracteres?

- ¿Qué contexto internacional, económico, político o social determina la transferencia de saberes de los@usuarios?

- ¿ ¿Cuáles son las estrategias lingüísticas que se manifiestan en las redes sociales?

El análisis del discurso es una disciplina enfocada en el contenido semántico de los términos usados en determinados textos, a su vez insertados en un contexto social, político o cultural. Proviene, por un lado, del estructuralismo norteamericano, a través de los seguidores de la obra de Zellig Harris (1954), y, por otro lado, de la escuela francesa de análisis del discurso a partir de los estudios lingüísticos de Michel Pêcheux (1975), Dominique Maingueneau (1976) y la lingüística del texto (Halliday \& Hasan, 1976).

Los aportes de Michel Foucault desde las ciencias sociales participaron en la constitución del análisis del discurso. El estudioso francés introdujo la línea del discurso en el análisis lingüístico al indagar sobre las relaciones entre poder, conocimiento y texto. En sus obras Vigilar y castigar (1976) y La voluntad del saber (1981) denuncia las aristas del poder a través de las manifestaciones, formas e instituciones en las que se puede basar un discurso. Luego, en La arqueología del saber (1999), Foucault se enfoca en el historicismo de los textos y su construcción social en una esfera discursiva para determinar ciertas zonas de prohibición, censura y tabúes.

Los lingüistas Luis Cortés Rodríguez y María Matilde Camacho Adarve aportaron en 2003 una obra clarificadora, ¿Qué es el análisis del discurso?, donde retoman los postulados de Benveniste, Jakobson, Bajtín y Halliday, visto que ellos sitúan al objeto de estudio no en la oración o el lenguaje, sino en las funciones del discurso. Hacia la constitución de una nueva disciplina se dirigen los compiladores Ruth Wodak y Michael Meyer en la obra Métodos de análisis crítico del discurso (2003). Esta versión en español forma parte de la colección Lingüística, Análisis del Discurso, que cuenta con tres obras más de Teun van Dijk, dos de ellas como compilador, El discurso como estructura y proceso (2000) y El discurso como interacción social (2000). Y aunque esta perspectiva tiene importantes fundadores 
pertenecientes a Europa y Asia, en América Latina no ha pasado desapercibida gracias a los estudios, entre otros, de Berardi (2003), Bolívar (2004) y Magalhaes (2005).

La vinculación del poder y la ideología aparece reflejada en las investigaciones de Norman Fairclough, quien centra su interés en el análisis del discurso orientado textualmente (Londoño \& Bermúdez, 2013). Para él, el este "es una teoría, o un método, que está en una relación dialógica con otras teorías y métodos sociales, los cuales deben engranar en una transdisciplinariedad más que en una forma interdisciplinaria" (2001, p. 121). Este enfoque considera el discurso como práctica social, que puede ser transgredida y a su vez está constituida por otros sistemas de lenguajes, de modo que resulta imprescindible una búsqueda semiótica para entender por ejemplo las relaciones entre el discurso, la gramática, la morfosintaxis, los medios de comunicación y los públicos.

Por otro lado, los estudios cognitivos propuestos por Van Dijk consideran el ACD como un enfoque multidisciplinario que incluye la lingüística, la pragmática y la semiótica. Su propuesta tiene en cuenta la micro-, la macro- y la superestructura en el diálogo texto-contexto del conocimiento. Aquí se analizan la sintaxis de cada oración que conforma un párrafo, el tema como eje transversal de un texto y la forma, como una manera de marcar un estilo determinado. En este caso, el discurso es un evento comunicativo amplio, con una fuerte carga ideológica (Van Dijk, 2014). Estos últimos enfoques, el análisis del discurso orientado textualmente de Fairclough y los estudios cognitivos de Van Dijk, muestran una relación dialógica entre el poder, la ideología, el conocimiento y la semiótica y permiten un análisis de los círculos de dominación y los dominados, junto con sus formas más extendidas.

Los especialistas en $\mathrm{AD}$ han suplido la carencia epistemológica de la teoría fundamentada en el discurso de los medios, y esta discusión ya ha alcanzado el universo de los social media. La perspectiva lingüística de ACD ha sido eficaz en la investigación de cómo los movimientos sociales y grupos de interés protestan y resisten contra la opresión de los abusos de poder (Chiluwa, 2012) y en la manera en que las crisis socioeconómicas transgre- 
den los límites políticos para ocupar el espacio de los social media mediante un proceso de resemántica y reescritura (Georgakopoulou, 2014).Vinculando estas nociones desde el universo de las redes sociales, es precisa la elaboración de un instrumento metodológico que explicaremos a continuación.

\section{Referentes metodológicos}

Para la concreción de este trabajo, aplicamos el ACD. El estudio inicia con la selección de una muestra dirigida de los mensajes etiquetados con \#5DaysForTheFive, de modo que se establece al tuit como unidad de análisis y práctica discursiva. A partir del comienzo de la Tercera Jornada 5 días por los 5, efectuada en Washington, D. C., tomamos como universo de muestra la semana comprendida desde el 4 al 11 de junio de 2014, donde se enmarcaba la actividad internacional.

La selección de los mensajes se realizó a través de la aplicación de escritorio Stela para la minería de redes sociales, que forma parte de un proyecto de investigación mayor realizado por un equipo de informáticos de la empresa Datys Tecnología \& Sistemas, ${ }^{9}$ división sistema de gestión de contenidos, en Santiago de Cuba.

El software Stela se ocupa de recuperar la información generada en Twitter, online, luego de siete días después de un hecho, a partir de diversos criterios que se pueden ampliar o reducir de acuerdo con las necesidades de los investigadores y el tema en cuestión. Nos referimos a las palabras clave, el uso de las almohadillas y las direcciones electrónicas, el idioma de los mensajes y si estos han sido emitidos desde o para una cuenta en particular. Por otro lado, la aplicación identifica algunas estadísticas dentro de un tema global específico que están dadas por los mensajes negativos, neutros y positivos, los principales usuarios que participan en los trending topics y la mayor cantidad de mensajes publicados en un día, entre otros criterios.

$\mathrm{Al}$ asumir como macroestructura semántica o significado global el tema de los cinco cubanos, a partir de la selección de la etiqueta \#5DaysForTheFive, tenemos en cuenta los significados locales que determinan los

9 Para más información, consúltese: http://www.datys.cu 
temas globales. Tales funciones se evidencian en los tuits escritos durante la producción y la comprensión del discurso hecha por los usuarios en un contexto dado. Pero como el ACD implica la presuposición a veces indirecta mediante un análisis estilístico y semántico sutil de la información que no aparece en los mensajes, relacionamos una búsqueda ideológica que ofrecerá una panorámica desde el otro lado de los mensajes. De alguna manera, este estudio sobre la etiqueta \#5DaysForTheFive traza una línea perspicaz con el análisis de audiencias digitales relacionadas con este tópico que amerita otros momentos investigativos, pues entran en juego fuerzas sociales tan complejas como el imaginario de los dominados y los discursos de reproducción de las prácticas de dominación, que deberían incluir otras metodologías en un campo también multidisciplinario.

Durante el periodo que constituye el objeto de estudio, se pudieron almacenar 8317 mensajes, escritos en los idiomas español e inglés, con la almohadilla \#5DaysForTheFive. De este universo consideramos la polarización de grupo de pertenencia y grupo ajeno para clasificar los mensajes que contenían palabras o \#etiquetas como "terror", "terrorismo", "terrorista”; "EE. UU.” y “Cuba”; "agentes”, “espías”, “espionaje” y "héroes”; "Obama” o "Barack Obama”, y "Castro" o "Fidel Castro", se compilaron un total de 3487 tuits, suficientes para nuestro objetivo, a los que se les aplicó el instrumento metodológico.

La metodología empleada es el análisis del discurso, basándonos en la actitud del ACD a partir de las nociones de los estudios sociocognitivos y el análisis de discurso orientado textualmente. Estos enfoques aplicados al universo de las redes sociales, en especial a Twitter, han favorecido la construcción de un método basado en sus especificidades de texto, contexto, ideología y semiótica, y se ha asumido una perspectiva transdisciplinar de investigación propia para el caso que se analiza.

Luego de la selección y posterior clasificación, el instrumento elaborado tiene en cuenta dos grandes etapas: la organización discursiva y la interpretación semiótico-discursiva. 
La organización discursiva comprende el análisis de la morfosintaxis a través de los elementos que constituyen parte de las oraciones presentes en los tuits. Aquí se tienen en cuenta la gramática, los elementos lingüísticos y paralingüísticos constitutivos de los mensajes y su coherencia y organización dentro de la oración. En cada una de estas estructuras se analizarán las proposiciones a partir del uso de argumentos, las nominalizaciones, los verbos modales o auxiliares, los adverbios y las características de los predicados.

Paralelo a una búsqueda sintáctica, se tendrán en cuenta los elementos estilísticos y retóricos que orientan los individuos o agentes del conocimiento a través de diversos roles. Estas figuras retóricas nos permitirán descubrir las persuasiones evidentes en los tuits para enfatizar o resaltar alguna práctica discursiva.

El análisis de la macroestructura (los temas) presentes además en los mensajes permitirá una búsqueda mucho más centrada en la característica social de este evento comunicativo. Aquí se estudiarán los actores del discurso y sus grados de abstracción, implicaciones, metáforas y otras estructuras semióticas.

La segunda etapa del instrumento metodológico, la interpretación semiótico-discursiva, comprende el análisis estadístico desde la aplicación del software Stela. Se tendrán en cuenta los reportes de sentimientos positivos, negativos y neutrales, que están determinados por las posiciones políticas e ideológicas que asumen los prosumidores al escribir mensajes con la etiqueta \#5DaysForTheFive.

En Stela se utiliza el algoritmo no supervisado SAHO para la detección de polaridades. ${ }^{10}$ Este proceso consiste en limpiar el texto del tuit de las direcciones electrónicas, letras repetidas y los símbolos de las almohadillas (\#) y el reemplazo de los emoticones por palabras o conjuntos de palabras. Luego se aplica una suma de un valor asociado a la palabra en un corpus que puede ser positiva, muy negativa, negativa, muy negativa o

10 El método no supervisado para la clasificación de polaridad en Twitter ya transita por su segunda versión. Para más información sobre el primer estudio realizado por los investigadores Reynier Ortega, Adrián Fonseca, Yoan Gutiérrez y Adrián Montoyo (2013), consúltese: http://aclweb.org/anthology/S/S13/S13-2083.pdf 
neutra y se le asigna la polaridad ( $>0$ positivo, 0 neutral, $<0$ negativa) asociada al valor resultante de la suma.

También se obtienen los datos de los usuarios más participativos por su actividad de tuitear o retuitear mensajes en el evento, y de los más influyentes, que se obtienen por la cantidad de seguidores con cuentas reales que aparecen en la red de microblogging.

Otras estadísticas muestran los reportes de las almohadillas más empleadas en los mensajes, además de la sugerida por el evento comunicativo, \#5DaysForTheFive, un mapa conformado por las geolocalizaciones de los autores de los tuits y, por último, los usuarios y las direcciones electrónicas más referenciadas en el marco de 140 caracteres.

La valoración hermenéutica de esta información, que se presentará en forma de gráfico (figura 1)., sobre lo que está escrito y lo que está ausente, ayudará a comprender los encuadres discursivos de héroes, espías o agentes que los medios han socializado en las personas en torno al caso de los cinco cubanos, y la manera en que los grupos dominados se enfrentan a tales proposiciones y socializan otras pertenecientes a su imaginario.

La segunda parte de la interpretación semiótico-discursiva se orienta a partir del análisis ideológico, donde se demostrarán con ejemplos los valores de concretización y comparación negativas. Se tendrán en cuenta los grados de abstracción evidenciados en la generalización de argumentos, la polarización nosotros/ellos, los móviles de compasión, las advertencias y las presuposiciones. Para una mayor comprensión del instrumento, elaboramos la tabla 1 .

\section{Análisis de resultados}

Luego del análisis discursivo de la muestra de 3487 mensajes etiquetados con \#5DaysForTheFive, detectamos una serie de señales simbólicas contentivas de ideología, poder y dominación, que determinan los encuadres de "héroes, espías o terroristas" socializados por los medios de comunicación en torno al evento comunicativo de los cinco cubanos y a su vez re- 


\section{Figura 1}

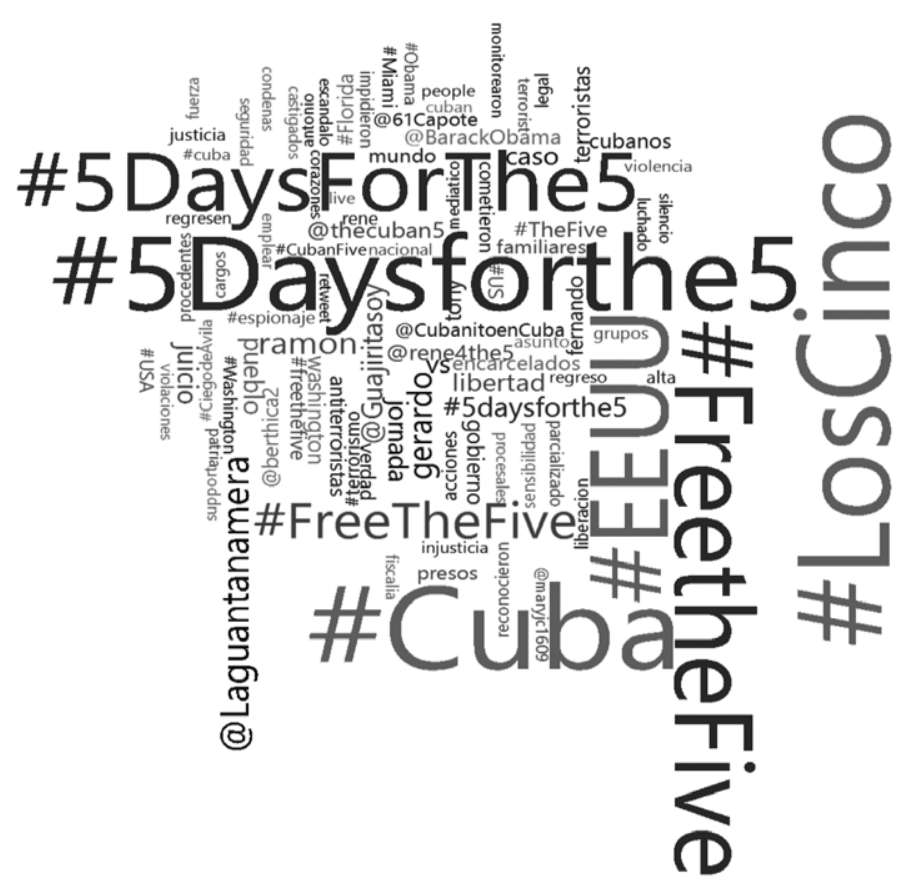

Tabla 1

\begin{tabular}{|c|c|}
\hline \multicolumn{2}{|c|}{ Selección y clasificación de los tuits } \\
\hline Organización discursiva & Interpretación semiótico-discursiva \\
\hline 1. Análisis morfosintáctico & 1. Análisis estadístico \\
\hline $\begin{array}{l}\text { - Gramática, elementos lingüísticos y paralingüísticos, } \\
\text { coherencia y organización en la frase. } \\
\text { - Proposiciones: argumentación, nominalización, verbos } \\
\text { modales o auxiliares, adverbios, características de los } \\
\text { predicados. } \\
\text { - Elementos estilísticos y retóricos. }\end{array}$ & $\begin{array}{l}\text { - Reporte de sentimientos positivos, negativos y neutrales. } \\
\text { - Usuarios más participativos, más influyentes y más } \\
\text { referenciados; localizaciones por usuarios. } \\
\text { - Almohadillas más empleadas y direcciones electrónicas } \\
\text { más referenciadas. }\end{array}$ \\
\hline 2. Análisis temático (macroestructuras) & 2. Análisis ideológico \\
\hline $\begin{array}{l}\text { - Temas y significados locales. } \\
\text { - Actores informativos (usuarios, instituciones, grupos, } \\
\text { partidos políticos. } \\
\text { - Implicaciones discursivas, metáforas. }\end{array}$ & $\begin{array}{l}\text { - Valores de concretización, comparación negativa. } \\
\text { - La polarización nosotros/ellos, los móviles de compasión, } \\
\text { las advertencias y las presuposiciones. }\end{array}$ \\
\hline \multicolumn{2}{|c|}{ Interpretación de datos } \\
\hline \multicolumn{2}{|c|}{ Propuesta de conclusiones } \\
\hline
\end{tabular}


marcados en las redes sociales. Un ejemplo de los usuarios, almohadillas y palabras clave más influyentes se evidencia a través de la nube de términos que presentamos a continuación:

Comencemos por la primera etapa, la "Organización discursiva”, a través del análisis morfosintáctico de los elementos antes descritos y de acuerdo con las construcciones cognoscitivas de 140 caracteres, evidentes en la muestra discursiva de \#5DaysForTheFive. Gramaticalmente se emiten juicios y valoraciones con el uso de oraciones activas donde se representa a los responsables de los hechos. @guajiritasoy escribe: "Juicio contra \#LosCinco en \#Miami NO tuvo garantías procesales, fue manipulado por los anticubanos de la \#Florida \#5DaysForThe5 \#Cuba \#EEUU”, mientras que @reynaturro dice: "We must intensify the battle for the return of Gerardo, Ramon and Antonio to \#Cuba as soon as possible \#5Daysforthe5 \#FreetheFive".

En estos formatos, ambos usuarios describen dos acciones que repercuten en el caso político. En el primero se representa a "los anticubanos de la Florida" como los culpables del resultado del proceso, que además careció de garantías, y el uso del adverbio de negación en mayúsculas remarca el hecho precedente. La voz "anticubanos" revela determinados conocimientos socialmente compartidos, donde intervienen cubanos radicados en la Florida y en contra del sistema que impera en la isla. En el segundo caso, su autor manifiesta la responsabilidad de la acción en "nosotros" y utiliza un verbo activo como "intensificar" para denotar mayor actividad, que luego queda aclarado en el tiempo gracias a la expresión adverbial "tan pronto como sea posible".

Otros usuarios de Twitter, en revancha, aprovechan el contexto sociopolítico internacional en Estados Unidos para describir acciones paralelas que tienen lugar en Cuba. @ErMachette retuiteó una nota del sitio @CubanetNoticias: “\#Cuba Aparece degollado en Guantánamo simpatizante de UNPACU \#5DaysForTheFive http://t.co/ecOgha5XIz La organización opositora, c... h...”. Aquí se retoman hechos concurrentes en el tiempo que contrarrestan la dominación ejercida por los medios en un intento de ocultar tales acontecimientos, los mismos que aparecen en la dirección electrónica incluida en el mensaje. 
Aparecen en los mensajes, asimismo, omisiones de letras y frases, faltas de ortografía y algunas direcciones electrónicas que amplían o envían a los lectores de los tuits a otros temas relacionados en el texto. Esta característica recalca la construcción de una nueva síntesis discursiva enmarcada en las proposiciones de la red social de microblogging, donde simples oraciones relacionan eventos comunicativos.

Para un análisis lexical tomemos los tuits enviados por @yoelexposito: "El juicio vs \#LosCinco se realizó en \#EEUU en un clima de hostilidad extrema, sin garantías procesales \#FreetheFive” y la usuaria @angelesdeabril, cuando dice: "Gobierno de \#EEUU efectuó pagos secretos a periodistas para que crearan una imagen de culpabilidad de \#LosCinco \#5Daysforthe 5 \#FreetheFive”. @Guajiritasoy también escribió: “Tony, Ramón y Gerardo presos en \#EEUU por ANTITERRORISTAS, mientras \#PosadaCarriles sigue libre en \#Miami por TERRORISTA".

De acuerdo con estos enfoques, es abundante la información que tienen los usuarios en torno al caso y son varias las unidades léxicas que emplean. La situación descrita primeramente como "un clima de hostilidad extrema" constituye una hipérbole, al calificar de esta manera los hechos ocurridos. En el segundo mensaje se aportan elementos argumentativos como "los pagos secretos a periodistas" para aumentar la culpabilidad de los cinco cubanos, al menos desde la prensa. Estas unidades léxicas ayudan a describir a los participantes en los hechos y los objetivos que tenían (juicio, gobierno, periodistas), además de que revelan los modelos mentales que utilizan las personas para comprender los acontecimientos y las ideologías a las cuales pertenecen.

Por su parte, en el tercer mensaje aparece la comparación entre nuestras buenas acciones y sus malas acciones y de manera lexical y gramatical se remarcan ciertos argumentos. Tony, Ramón y Gerardo son antiterroristas y Posada Carriles es terrorista, en un mismo país, aunque con diferentes significados, como se describe en el mensaje a Estados Unidos y Miami. Los conceptos que para un grupo constituyen "terroristas" para otros son "héroes o mártires" y ambas nociones son específicas desde el uso de la mayúscula. 
Estos mensajes aquí analizados constituyen una muestra del conocimiento extendido y socializado entre las comunidades en pugna alrededor del caso de los cinco cubanos y de la forma en que responden discursivamente a la dominación ejercida por la prensa que da cobertura a los hechos. Las opiniones emitidas por los usuarios denotan representaciones sesgadas de la realidad, dada la ausencia de elementos cuantificadores y realmente notables en la participación discursiva.

En varios de los mensajes se percibe igualmente el uso de elementos retóricos que, si bien carecen de un contenido cognitivo, demuestran un grado de sensibilidad y muestra afectiva. Los usuarios @fonseca julio y@Laguantanamera escribieron respectivamente: "Ola de solidaridad con \#Cuba en \#Toronto por \#losCinco http://t.co/Bs6Fn8ZhQp \#Ottawa \#Washington \#Miami \#PRico \#5DaysForThe5" y "Quedan tres corazones encarcelados sufriendo la separación de \#Cuba y sus familiares \#FreetheFive \# 5Daysforthe5”.

La expresión metafórica "una ola de solidaridad” en el primer tuit y el empleo del símil en el segundo a través del sintagma "tres corazones" son premisas que denotan la manera en que los usuarios corporizan sus ideologías y sus conocimientos sobre el mundo que los rodea, aunque también son nociones parciales del hecho en general.

Semánticamente se pone de manifiesto la respuesta que dan las comunidades desde las redes sociales a los discursos de poder y abuso del mismo a los que son sometidos. @yhonnyg dice: “\#LosCinco cubanos monitoreaban probadas organizaciones terroristas anticubanas en \#EEUU \#FreetheFive\#5Daysforthe5”.Y@450Amador manifestó: “\#LosCinco son héroes, no terroristas \#FreeTheFive \#5DaysForThe5 @jscomunicadores @Jupresidente \#Nicaragua \#Cuba http://t.co/dnealphHBt”. En cambio, @Ipaz1 1 declara que: "Fiscalía ocultó toda evidencia sobre actos terroristas vs \#Cuba \#FreetheFive \#5Daysforthe5”. A través de este estilo se mitiga la acción de los cinco cubanos en Estados Unidos al "monitorear probadas organizaciones", y en el segundo mensaje es evidente la acusación que se le hace a la Fiscalía por ocultar los datos sobre los actos terroristas. 
En este primer análisis morfosintáctico, son evidentes los abusos de poder y de conocimientos a los que son sometidas las comunidades de usuarios que responden a través de la red de microblogging Twitter. Se perciben opiniones sesgadas y una comparación entre buenos y malos a partir de las ideologías compartidas y las situaciones sociales que rodearon los hechos.

Pasemos al análisis temático de la muestra discursiva de \#5DaysForTheFive. En varios de los mensajes seleccionados aparecen los significados locales y los actores desde sus amplios grados cognoscitivos. Por ejemplo, @val6ntina y@JJoHerrera escriben: “@visiondesdecuba: Buenos dias.. iYa le dijiste a \#Obama q es hora de poner \#FinALaInjusticia? \#5DaysForThe5 \#FreeTheFive" y "Obama no liberará a \#LosCinco si no es por una gran movilización internacional y especialmente del pueblo \#EEUU \#FreetheFiv...”.

Dentro de la macroestructura temática evidenciada en el caso de los cinco, aparecen otros términos locales como injusticia y libertad, los cuales son reconocidos por las comunidades que participan desde Twitter en este evento comunicativo.

Otros dos mensajes, emitidos por@Jaime48882425y@Jotaviz dicen: "\#Cuba: \#EEUU debería atender la voluntad de acercamiento de su \#sociedad http://t.co/zsyUMdiXet @PortalCubasi \#5DaysForThe5 \#5days4cuban5” " “\#Cuba. Hace 2 años \#UNPACU es la organización opositores con mas presos políticos \#5DaysForTheFive”. Nótese en estos dos tuits la naturaleza de las representaciones locales en ambos contextos políticos y sociales, y la forma en que se responde discursivamente a la dominación ejercida por la prensa de ambos países.

En estos formatos también son claros los actores participantes y sus grados de abstracción. Se mencionan el presidente y el pueblo de Estados Unidos, así como Cuba y la sociedad cubana, y los roles que deben desempeñar para la resolución de los conflictos políticos que los relacionan. Aquí tienen tanto poder de pensamiento y ejecución el pueblo como los presidentes, visto que ambos forman parte de un mismo contexto social. 
El análisis temático apunta hacia los temas locales que son remarcados ideológicamente desde el discurso y los responsables de llevarlos a cabo, desde sus funciones e identidades. Se remarca la polarización entre los grupos nosotros/ellos, así como la identificación de sus valores y prejuicios.

Como conclusión de esta primera etapa, "organización discursiva”, se percibe desde la búsqueda lingüística de los mensajes y el análisis de los temas y actores informativos abusos de conocimiento y poder relacionados con el caso de los cinco cubanos, dados por las respuestas que emiten los usuarios de Twitter. Esta impronta se socializa gracias a la influencia y los encuadres discursivos realizados por la prensa norteamericana y cubana y otras instituciones epistémicas para orientar la opinión pública.

Pasemos a la segunda etapa, la "interpretación semiótico-discursiva”, desde los exámenes estadístico e ideológico. Una de las descripciones seleccionadas luego de aplicar el software Stela en el tratamiento de la muestra de 3487 tuits en torno al evento comunicativo de \#5DaysForTheFive, estuvo en el análisis de sentimientos, que quedó definido como aparece en la figura 2.

De la muestra, 1251 tuits constituyen mensajes positivos, lo que equivale a un $35,88 \%$. Este resultado es previsible porque el caso que se analiza es un evento de solidaridad, anunciado así desde la misma convocatoria. Sin embargo, 1391 neutrales y 829 negativos, que representan el 39,88\% y

\section{Figura 2}

\section{Análisis de sentimientos}

\section{Estadísticas por orígenes de datos}

\begin{tabular}{|c|c|c|c|}
\hline \multicolumn{4}{|l|}{ Prueba } \\
\hline \multicolumn{3}{|c|}{$6.01 / 10$} & \\
\hline Positivos: & 1251 & $35.88 \%$ & Negativo \\
\hline Negativos: & 829 & $23.77 \%$ & Neutral \\
\hline Neutrales: & 1391 & $39.89 \%$ & No Procesado \\
\hline No procesados: & 16 & $0.46 \%$ & Positivo \\
\hline Total: & 3487 & & \\
\hline
\end{tabular}


el $23,77 \%$, respectivamente, dan fe de otras respuestas que en materia discursiva emiten los usuarios sobre el caso de los cinco y sobre otras temáticas relacionadas. La determinación de los sentimientos a partir de los contenidos ideológicos que emiten los usuarios y sus seguidores en la red social quedaría trunca si no se tienen en cuenta los orígenes de las cuentas, la naturaleza de los retuits y los sitios y las personas más influyentes dentro del evento comunicativo que se describe. La figura 3 ilustra otras estadísticas importantes para este estudio.

\section{Figura 3}

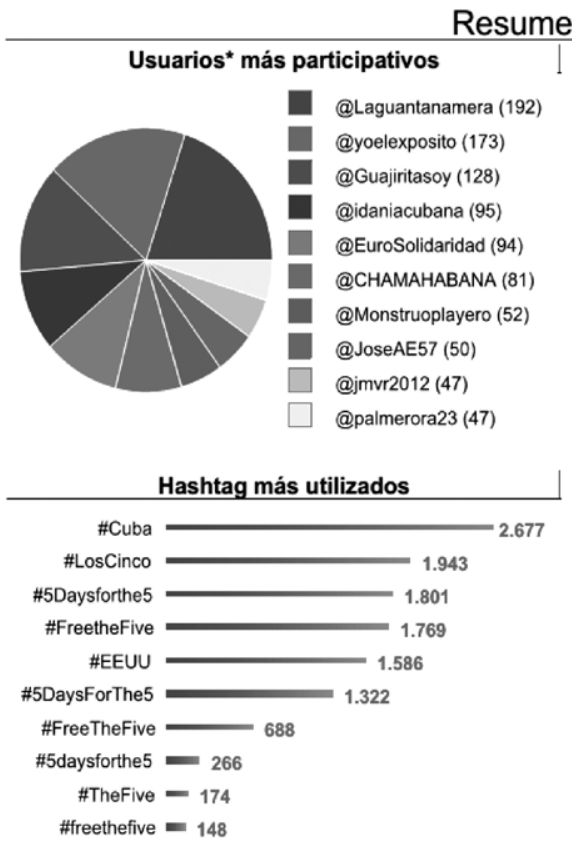

\begin{tabular}{lc}
\multicolumn{2}{c}{ Usuarios* más influyentes } \\
\hline Usuarios & Seguidores \\
\hline @lzarraDeVerdad & 542,876 \\
@JesusFariaPSUV & 72,505 \\
@gabrieladelmarp & 64,649 \\
@CubanitoenCuba & 47,816 \\
@twizquierda & 38,319 \\
@JuventudRebelde & 27,209 \\
@Caninator & 25,015 \\
@CubaenNoticias & 22,339 \\
@yeidckol & 22,094 \\
@freecuban5 & 21,337
\end{tabular}
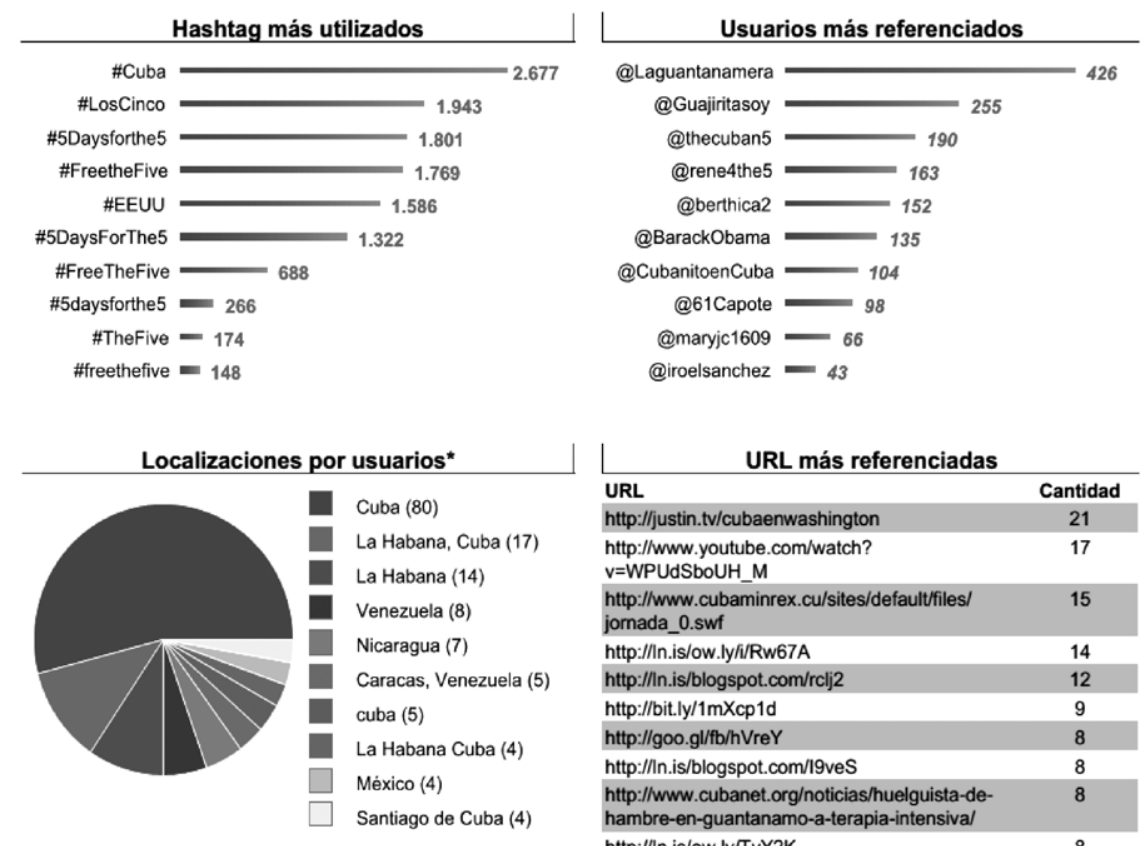

\begin{tabular}{|c|c|}
\hline \multicolumn{2}{|l|}{ URL más referenciadas } \\
\hline URL & Cantidad \\
\hline http://justin.tv/cubaenwashington & 21 \\
\hline $\begin{array}{l}\text { http://www.youtube.com/watch? } \\
\text { v=WPUdSboUH_M }\end{array}$ & 17 \\
\hline $\begin{array}{l}\text { http://www.cubaminrex.cu/sites/default/files/ } \\
\text { jornada_0.swf }\end{array}$ & 15 \\
\hline http://ln.is/ow.ly/i/Rw67A & 14 \\
\hline http://ln.is/blogspot.com/rclj2 & 12 \\
\hline http://bit.ly/1mXcp1d & 9 \\
\hline http://goo.gl/fb/hVreY & 8 \\
\hline http://nn.is/blogspot.com/l9veS & 8 \\
\hline $\begin{array}{l}\text { http://www.cubanet.org/noticias/huelguista-de- } \\
\text { hambre-en-guantanamo-a-terapia-intensiva/ }\end{array}$ & 8 \\
\hline http://ln.is/ow.ly/TvY2K & 8 \\
\hline
\end{tabular}

* Usuarios autores de tweets. 
Como se constata, la mayoría de los usuarios, como participantes, influyentes o referenciados, y sus localizaciones están dentro de los límites mediáticos cubanos. Esta característica alude a la legitimación discursiva de "héroes" que ha tenido el caso de los cinco en Cuba, junto con las formas simbólicas en que se han socializado los abusos de poder. Aunque el evento político de solidaridad \#5DaysForTheFive, tuvo lugar en Washington, la mayor cantidad de mensajes fueron emitidos principalmente desde La Habana y países como México, Venezuela y Nicaragua. Se trata de prosumidores cubanos que participan desde y con \#Cuba respondiendo a los abusos de poder alrededor de la información y el conocimiento. También participan otros usuarios europeos que, si bien son los menos, no dejan de ser importantes por los resúmenes que aportan.

Como segundo examen dentro de la "interpretación semiótica-discursiva”, corresponde el análisis ideológico, donde se han detectado señales discursivas de concretización. Estudiemos algunos mensajes, como los que escriben @maryjc1609 y @LibradoLinares: "sí, rompamos el cerco!! @JulietaCT Deben conocer que en \#EEUU se comete esta atroz injusticia \#5DaysForThe5 http://t.co/9NG...” y“\#CUBA 7 actvs d MCR-UNPACU y FRN-OZT hacen acción civica (pancarta, bandera nac y arenga) x cese represion contra opo...\#5DaysForThe 5". En ambos enfoques se describen, con acciones prácticamente visuales -"esta atroz injusticia” y "pancarta, bandera, arenga" -, los actos negativos de uno y otro grupo, además de incluirse las direcciones electrónicas donde se amplía el contenido de los mensajes.

También encontramos otros usuarios que hacen uso semánticamente de la presuposición al describir la naturaleza del caso que se analiza. @61Capote escribe: "but \#cuba have @thecuban5 who are symbol for world resistance \& struggle against capitalism \#5DaysForThe5”. Los símbolos de "resistencia mundial y lucha contra el capitalismo" se hacen prácticamente visibles en el ejemplo demostrado por el caso de los cinco cubanos. Esta es una característica ideológica para socializar conocimientos ante las comunidades virtuales de las redes sociales.

La polarización ideológica de grupo de pertenencia y grupo ajeno se manifiesta en varios mensajes. @AlinePrezNeri escribe: "El mundo gri- 
ta ¡fin a la injusticia, libera a los \#Cinco! pero \#Obama hace como que no escucha \#5DaysForThe5”. El sustantivo “mundo" resume aquí las diversas acciones solidarias que tienen lugar por la liberación de los cinco cubanos, mientras que el presidente norteamericano permanece callado. Luego, el usuario@ObamaFreeThe5 dice: “@BarackObama Yes You Can Free All The \#CubanFive Now! \#US political prisoners \#5DaysForThe5 \#Cuban5 \#LosCinco \#Cuba ht...."

Nótese la amplia responsabilidad que se le otorga al mandatario de Estados Unidos, en este caso particularmente. Sin embargo, los juicios de polarización también difieren a partir de los buenos y los malos, en relación con los usuarios y los contrastes por oposición. El mensaje de @JorgeLu66794149 califica como una comparación negativa: "GErardo NO es ASESINO http://t.co/8SvTdcilay \#5DaysForThe5 \#Cuba \#EEUU \#FreeTheFive. El ASESINO es \#PosadaCarriles y es...".

Por otro lado, @jdanielferrer arremete una vez más comparando el caso de los cinco con otra organización: “\#Cuba. \#UNPACU es la organización que mas agentes infiltrados he desenmascarado en la historia de la oposición \#5DaysForThe5”. La comparación de ambos sitúa la responsabilidad tanto en las personas enjuiciadas, como en los lugares donde se desenmascara la dominación discursiva. De esta segunda etapa, "interpretación semiótico-discursiva”, en la muestra analizada del evento \#5DaysForTheFive, detectamos que los abusos de poder e información sobre el caso de los cinco están determinados por los conocimientos socialmente compartidos entre las comunidades, la mayoría de ellos aportados por la prensa referida a los cubanos.

\section{Conclusiones}

Desde el discurso tuiteado y retuiteado en un evento comunicativo de solidaridad como \#5DaysForTheFive, los cinco cubanos pueden ser héroes, agentes o espías. Esta característica es consecuencia de la naturaleza de los encuadres periodístico, político e ideológico socializados por la prensa norteamericana y cubana. @Guajiritasoy, uno de los usuarios más participativos, con 128 mensajes, y referenciado en al menos 255, de una muestra 
de 3487 mensajes analizados, es un ejemplo de cómo los actores copian en las redes sociales públicas un discurso periodístico específico cuando escribe “Tony, Ramón y Gerardo presos en \#EEUU por ANTITERRORISTAS, mientras \#PosadaCarriles sigue libre en \#Miami por TERRORISTA”.

Otros usuarios de la red de microblogging, @patrycuba151279 y @ yeidckol, manifiestan varios conocimientos, dada la influencia de la prensa: "We defend our heroes! \#TheFive have been victim of cruel, inhumane and degrading treatment and punishments \#FreetheFive \#5Daysforthe5 \#Cuba" y "Esperamos que la jornada en Washington \#5daysforthe5 sea un paso más para logra la libertad de 3 héroes. \#freethefive \#Cuba @...”.

Hay que tener en cuenta desde qué lado se emiten y en qué lado se analizan los juicios, las calificaciones y los elementos semiótico-discursivos, o sea, los contextos de producción y consumo discursivos. Además, son importantes los contextos políticos, sociales y lingüísticos que rodean a los eventos comunicativos, junto con los conocimientos que poseen los prosumidores y la manera en que ellos dan respuesta a las formas de dominación o abuso simbólicos a los que son sometidos.

Con este estudio se corrobora que las relaciones de poder, ideología y dominación están presentes en las formas en que los usuarios responden a ellas, al menos desde las redes sociales. También se comprueba la pertinencia de las actitudes del análisis crítico del discurso para la investigación de texto y contexto sobre las cibercomunidades de Twitter. Finalmente, se hace evidente que la dominación y el abuso de poder y de la información en torno al caso de los cinco cubanos tienen lugar, en gran medida, gracias a la legitimación de este desde el discurso, pero desde el discurso político socializado en Cuba.

Esta tercera hipótesis se comprueba en los ejemplos ofrecidos por los usuarios de Twitter y en el análisis de los contenidos y juicios que emiten, a partir de los modelos mentales establecidos anteriormente. @CHAMAHABANA, con una participación en 81 mensajes, dice: “\#Venezuela \#Argentina \#Brasil \#Miami \#EEUU \#Cuba \#FreeTheFive \#5DaysForThe5 
GERARDO, TONYY ANTONIO SON HEROES CUBANOS PRESOS INJUSTAMENTE”. @61Capote, referenciado en 98 mensajes, también escribe: "\#LosCinco no son espías, no fueron enjuiciados por \#espionaje \#FreetheFive \#5Daysforthe5”.

Si bien el discurso per se no puede dar solución a los conflictos políticos y beligerantes entre países, como en el tema que nos ha ocupado, porque intervienen otros contextos económicos y sociales, al menos desde los social media como Twitter es posible que las comunidades se unan desde el conocimiento y aporten otros saberes que se puedan contraponer a la dominación simbólica que ejercen los monopolios de la prensa internacional. Mediante un tuit es posible cambiar la estrategia ideológica de abuso presente en el discurso, pero esta debe confluir política y socialmente, con los mismos códigos lingüísticos en que se hace la afrenta.

\section{Bibliografía}

Berardi, L. (2003). Análisis crítico del discurso: perspectivas latinoamericanas. Santiago: Frasis.

Bolívar, A. (2004). Análisis crítico del discurso de los académicos. Signos, $37(55), 7-18$.

Chiluwa, I. (2012). Social media networks and the discourse of resistance: A sociolinguistic CDA of Biafra online discourses. Discourse \& Society, 23(3), 217-244.

Conde López, J. \& Moreno Rey, F. (2012). De las ideas políticas a Twitter: el desbordamiento de la campaña electoral en la Democracia 2.0. Ponencia presentada en el I Congreso Internacional de Comunicación Política y Estrategias de Campaña "Comunicación Política 2.0 y Liderazgo". 6 y 7 de julio, Madrid.

Cortés Rodríguez, L. \& Camacho Adarve, M. M. (2003). ¿Qué es el análisis del discurso? Barcelona: Octaedro y Ediciones Universitarias de Barcelona. 
Eco, U. (1973). La estructura ausente: introducción a la semiótica. Barcelona: Lumen.

Eco, U. (1991). Semiótica y filosofía del lenguaje. São Paulo: Editora Ática S.A.

Fairclough, N. (2001). Critical discourse analysis as a method in social scientific research. En Wodak, R. \& Meyer, M. (Eds.), Methods of Critical Discourse Analysis (pp. 121-138). Londres: Sage.

Falcón, R. A. (2014). Cinco héroes prisioneros del Imperio. Cuba Debate. Recuperado el 3 de mayo de 2014 de http://www.cubadebate.cu/ opinion/2004/03/10/cinco-heroes-prisioneros-del-imperio/\#. VNjyrvmG-gZ

Flores, J. M. (2012). Convergencia de las redes sociales en Internet y en la telefonía móvil. Innovaciones periodísticas en las redes sociales. Cybermedia, CD-ROM. ISBN 13: 978-84-616-2195-8 :: 21-38

Foucault, M. (1976). Vigilar y castigar: nacimiento de la prisión. Madrid: Siglo XXI.

Foucault, M. (1981). La voluntad de saber. México: Siglo XXI.

Foucault, M. (1999). La arqueología del saber. México: Siglo XXI.

Fumero, A. (2011). Sobre redes, personas, contenidos y derechos en la Red. Cuadernos de Comunicación Evoca, 5, 41-45.

García Luis, J. (2013). Revolución, socialismo y periodismo. La prensa y los periodistas cubanos ante el siglo XXI. La Habana: Editorial Pablo de la Torriente Brau.

Georgakopoulou, A. (2014). Small stories transposition and social media: A micro-perspective on the "Greek crisis". Discourse \& Society, 25(4), 519-539. 
Haber Guerra, Y. (2014). ¿Tecnofilia, alternidad o pensamiento ácrata? Metamediación y periodismo complejo. Pauta Geral - Estudos em Jornalismo, 2(2), 3-21.

Halliday, J. A. K. \& Hasan, R. (1976). Cohesion in English.Londres: Longman.

Harris, Z. S. (1954). Distributional structure. Word, 10, 146-162.

Herzog, B., Tortojada, S., Agulló, V., Valderrama, J. C. \& Aleixandre, R. (2006). El análisis del discurso basado en la sociología del conocimiento como enfoque para la investigación sobre identidades. I Jornadas de Trabajo Medimigra, Universitat Jaume I.

Hjelmslev, L. (1943). Prolegomena to a theory oflanguague. California: University of Wisconsin Press.

Iluminati Lab (2012). 140 trending topics que hicieron historia. Elecciones México 2012, Social Media Intelligence.

Keller, R. (2005). Analysing Discourse. An Approach from the Sociology of Knowledge. Forum: Qualitative Social Research, 6(3). Recuperado el 14 de abril de 2014 de http://www.qualitative-research. net/fqs-texte/3-05/05-3-32-e.htm

Kimber, S. (2013). The Cuban five were fighting terrorism. Why did we put them in jail? The Washington Post. Recuperado el 25 de enero de 2014 de http://www.washingtonpost.com/opinions/ the-cuban-five-were-fighting-terrorism-why-did-we-put-themin-jail/2013/10/04/37c556a6-1fca-11e3-b7d1-7153ad47b549 story.html

Levy, E. I. (2013). ¿Cinco héroes o cinco espías? La verdad sobre el caso "Avispa" contada por uno de sus protagonistas. Elblog de Montaner. Recuperado el 25 de enero de 2014 de http: / / www.elblogdemontaner.com/cinco-heroes-o-cinco-espias-la-verdad-sobre-el-casoavispa-contada-por-uno-de-sus-protagonistas/ 
Londoño-Vásquez, D. A. \& Bermúdez-Restrepo, H. L. (2013). Tres enfoques sobre los estudios críticos del discurso en el examen de la dominación. Palabra Clave, 16(2), 491-519.

Londoño-Vásquez, D. A. \& Frías-Cano, L. Y. (2011). Análisis crítico del discurso y arqueología del saber: dos opciones de estudio de la sociedad. Palabra Clave, 14(1), 101-121.

Luz, M., Fernández, M. \& Moro, E. (2011). Twitter y política, información, opinión y ¿predicción? Cuadernos de Comunicación Evoca, 4, 11-15.

Magalhaes, I. (2005). Introducao: A Análise de Discurso Crítica. DELTA, 21, 1-9.

Maingueneau, D. (1976). Introducción a los métodos del análisis del discurso. Buenos Aires: Editorial Hachette.

Martin, L. \& Whittaker, R. (1998). Poder-Decir o el poder de los discursos. Madrid: Arrecife.

Martín Serrano, M. (2013). Mediaciones del conocimiento y de la información que humanizan y deshumanizan. Conferencia inaugural del VII Encuentro internacional de Investigadores y Estudiosos de la Información y la Comunicación. 28 de noviembre, Facultad de Comunicación, Universidad de La Habana.

Merodio, J. (2010). 365 días (y noches) de marketing 2.0. El inicio de la e-década. Madrid: Creative Commons. Autoedición.

Orihuela, J. L. (2011). Mundo Twitter: una guía para comprender y dominar la plataforma que cambió la red. Barcelona: Alianza Editorial.

Ortega, R., Fonseca, A., Gutiérrez, Y. \& Montoyo, A. (2013). SSA-UO: Unsupervised Twitter sentiment analysis. Second Joint Conference on 
Lexical and Computational Semantics ( ${ }^{*}$ SEM), 2. Seventh International Workshop on Semantic Evaluation (SemEval 2013), 501507. Atlanta, Georgia.

Pechêux, M. (1976). Les vérités de La Palice. Paris: Máspero.

Primera, M. (2014). Regresa a La Habana uno de los cinco espías cubanos presos en EE.UU. El País. Recuperado el 16 de mayo de 2014 de http://internacional.elpais.com/internacional/2014/02/28/actualidad/1393616282_952467.html

Público (2014). El 15-M vuelve a tomar las plazas en su tercer aniversario. Recuperado el 17 de mayo de 2014 de http://www.publico.es/ actualidad/15-m-vuelve-plazas-tercer.html

Sacks, H., Schegloff, E. \& Jefferson, G. (1974). A simplest systematic for the organization turn-taking in conversation. Language, 50, 696-735.

Stecher, A. (2010). El análisis crítico del discurso como herramienta de investigación psicosocial del mundo del trabajo. Discusiones desde América Latina. Universitas Psychologica, 9(1), 93-107.

Traxler, M. J. \& Gernsbacher, M. A. (2006). Handbook of psycholinguistics. Ámsterdam-Boston: Elsevier.

Turner, M. (1996). The literary mind. Oxford: University Press.

Van Dijk, T.A. (2000). El discurso como estructura y proceso. Barcelona: Paidós.

Van Dijk, T.A. (2000). El discurso como interacción social. Barcelona: Paidós.

Van Dijk, T. A. (2010). Discurso, conocimiento, poder y política: hacia un análisis crítico epistémico del discurso. Revista de Investigación Lingüística, 13, 167-215. 
Van Dijk, T. A. (2014). Discourse and knowledge. A sociocognitive approach. Cambridge: Cambridge University Press.

Wodak, R. (2003). De qué trata el análisis crítico del discurso. Resumen de su historia, sus conceptos fundamentales y sus desarrollos. En Wodak, R. \& Myer, M. (Eds.), Métodos de análisis crítico del discurso (pp. 17-34). Barcelona: Gedisa.

Wodak, R. \& Meyer, M. (2003). Métodos de análisis crítico del discurso. Barcelona: Gedisa. 